\title{
Die Deutsche Suchtstiftung Matthias Gottschaldt - Wilhelm-Feuerlein-Preis
}

$\mathrm{D}$ ie Deutsche Suchtstiftung Matthias Gottschaldt möchte durch einen intensiven Dialog zwischen Gesellschaft, Wissenschaft, Wirtschaft und Politik die Entstigmatisierung von Suchterkrankungen und die Anerkennung von Sucht als Krankheit in der Gesellschaft vorantreiben. Öffentlich geäußerte Ansichten über die Entstehung und Behandlung von Abhängigkeiten beruhen selten auf solidem Wissen. Massive Aufklärungsarbeit ist zu leisten und die Forschung auf diesem Gebiet zu unterstützen. Die Stiftung engagiert sich für die Idee einer gut informierten Wissensgesellschaft, in der ein verantwortungsvoller Umgang mit Abhängigkeiten Schritt für Schritt Wirklichkeit wird.

Die Deutsche Suchtstiftung Matthias Gottschaldt ist benannt nach dem Mann, der durch einen offensiven und ehrlichen Umgang mit seiner eigenen Alkoholabhängigkeit für viele ein positives Leitbild war und so die Akzeptanz dieser Erkrankung in der Gesellschaft erhöhte. Sucht war für ihn keine stigmatisierende Erkrankung, sondern eine Chance zur konstruktiven persönlichen Weiterentwicklung. Das Ziel, das die Stifterin Dr. Edda Gottschaldt mit der Deutschen Suchtstiftung verfolgt, ist es, jeden Einzelnen anzuregen, in kritischer Selbstverantwortung das eigene Verhalten im Umgang mit Genussmitteln ehrlich einzuschätzen und rechtzeitig Hilfe anzunehmen, wenn es geboten ist.

Um die Diskussion und den Erfahrungsaustausch unter Ärzten und Wissenschaftlern zu fördern, führt die Deutsche Suchtsstiftung in regelmäßigen Abständen eigene wissenschaftliche Symposien und Expertentagungen durch. Ein besonderer Fokus lag in den letzten Jahren auf der Unterstützung selbst abhängig erkrankter Ärzte. Hierzu wurde der „Expertenrat suchtgefährdete Ärztinnen und Ärzte“ gegründet. Ziel dieses Expertenrates ist es, ein Forum für alle Fragen der Prävention und Behandlung suchterkrankter Ärzte zu sein. Um genaue Informationen über den Umgang von potenziell abhängig machenden Substanzen zu erhalten, unterstützte die Deutsche Suchtstiftung Matthias Gottschaldt gemeinsam mit der Ärztekammer Hamburg eine bisher einmalige epidemiologische Totalerhebung aller Hamburger Ärzte im Praktikum (AIP) zu deren Substanzmittelgebrauch und Substanzmittelmissbrauch. Im Rahmen dieser Untersuchung konnte gezeigt werden, dass AIP's im Vergleich zur Allgemeinbevölkerung seltener einen riskanten Tabakkonsum, aber häufiger eine riskante Medikamenteneinnahme, insbesondere bei Schmerzmitteln aufweisen.

Mit dem Wilhelm-Feuerlein-Forschungspreis prämiert die Deutsche Suchtstiftung Matthias Gottschaldt besondere wissenschaftliche Leistungen auf dem Gebiet der Forschung über substanzgebundene Störungen, insbesondere im Bereich Alkohol und Medikamente. Der Wilhelm-Feuerlein-Forschungspreis wurde anlässlich des 10-jährigen Bestehens des Oberberg-Therapiemodells 1994 von Prof. Dr. Gottschaldt gestiftet und zunächst unter der Schirmherrschaft der DGPPN alle zwei Jahre verliehen. Seit Gründung der Deutschen Suchtstiftung Matthias Gottschaldt 1998 wird der Forschungspreis unter dem Dach der Stiftung verliehen. Der Preis ist mit 10000 Euro dotiert und wird auf Vorschlag des wissenschaftlichen Beirats, bestehend aus Prof. Böning, Prof. Heinz, Prof. Fritze, Prof. Ladewig, Prof. Mann, PD Dr. Mundle, Prof. Poser, Prof. Reimer und Prof. Schmidt, vergeben. Der Preis wird in der Regel geteilt in je eine Arbeit aus der Grundlagenforschung oder der Bevölkerungsepidemiologie und eine weitere Arbeit aus der Anwendungs- oder klinischen Forschung (incl. Versorgungsepidemiologie).

Im Rahmen der Serie „Deutsche Suchtstiftung Matthias Gottschaldt", die mit diesem Heft startet, sollen die diesjährigen Preisträger bzw. die prämierten Arbeitsgruppen vorgestellt werden. Dr. Tim Neumann als Vertreter der Arbeitsgruppe von Prof. Dr. Claudia Spies, Berlin, konnte in seiner randomisierten Untersuchung an verunfallten Patienten einer Rettungsstelle zeigen, dass es möglich ist, durch eine computergestützte Kurzintervention den Anteil der Patienten riskantem Alkoholkonsum zu senken. Im Bereich Grundlagenforschung wurde Priv.Doz. Dr. Gunter Schumann, Mannheim, für seine wissenschaftlichen Arbeiten zum Thema „Systematische Analyse der Bedeutung prä- und postsynaptischer glutamaterger Signaltransduktionsgene für die Alkoholabhängigkeit beim Menschen“ prämiert. In seiner Arbeit wird der Einfluss der Genetik auf die Entstehung einer Alkoholabhängigkeit beispielhaft dargestellt. Dipl.-Psych. Dr. Henning Krampe, als Vertreter der Arbeitsgruppe von Prof. Dr. Dr. Hannelore Ehrenreich, Göttingen, stellt die Ergebnisse der ambulanten Langzeit-Instensiv-Therapie für Alkoholkranke ALITA dar. Durch diese intensive Langzeittherapie mit täglichen Kurzkontakten ist es gelungen, die Gruppe der bisher als therapieresistenten Schwerstabhängigen zu erreichen und erfolgreich zu behandeln.

Die vorgestellten, mit dem Wilhelm-Feuerlein-Forschungspreis 2005 prämierten Arbeiten belegen das breite Spektrum der heutigen Suchtforschung auf international anerkanntem, qualitativ hohem Niveau. Die Deutsche Suchtstiftung Matthias Gottschaldt möchte durch die Prämierung dieser Arbeiten die Suchtforschung in Deutschland unterstützen. Durch die gesellschaftsrelevanten Aktivitäten sollen die Ergebnisse der Suchtforschung in die breite Öffentlichkeit getragen und die Anerkennung dieser Erkrankung erzielt werden.

\section{Dr. Edda Gottschaldt, Berlin \\ Priv.-Doz. Dr. Götz Mundle, Hornberg}

